livraisons

d'Histoire

de l'Architecture

\section{Livraisons de l'histoire de l'architecture}

$22 \mid 2011$

Émotions patrimoniales II

\title{
La destruction d'un lieu de mémoire, entre émotion et résistance
}

The Destruction of a Place of Memory, between Emotion and Resistance

Zerstörung einer Gedenkstätte, zwischen Emotion und Widerstand

\section{Françoise Clavairolle}

\section{(2) OpenEdition}

Journals

Édition électronique

URL : http://journals.openedition.org/lha/148

DOI : $10.4000 /$ /ha. 148

ISSN : 1960-5994

Éditeur

Association Livraisons d'histoire de l'architecture - LHA

Édition imprimée

Date de publication : 10 décembre 2011

Pagination : 21-32

ISSN : $1627-4970$

Référence électronique

Françoise Clavairolle, "La destruction d'un lieu de mémoire, entre émotion et résistance ", Livraisons de l'histoire de l'architecture [En ligne], 22 | 2011, mis en ligne le 10 décembre 2013, consulté le 19 avril 2019. URL : http://journals.openedition.org//ha/148 ; DOI : 10.4000/lha.148

Ce document a été généré automatiquement le 19 avril 2019

Tous droits réservés à l'Association LHA 


\section{La destruction d'un lieu de mémoire, entre émotion et résistance}

The Destruction of a Place of Memory, between Emotion and Resistance

Zerstörung einer Gedenkstätte, zwischen Emotion und Widerstand

\section{Françoise Clavairolle}

Le 11 juillet 2007, les forces de l'ordre se déploient dans la Vallée française (Lozère) dès les premières heures de la matinée. Sept fourgonnettes de gendarmes, des motards et une pelleteuse réquisitionnée gravissent le chemin abrupt qui conduit à la Picharlerie, un mas occupé depuis cinq ans par un groupe de squatters. Quelques heures plus tard, leurs effets personnels sont ensevelis sous un amas de pierres et de gravats de 50 mètres de long sur plusieurs mètres de large. (ill.1) 


\section{1 : Bâtiments délabrés en reconstruction. La Picharlerie occupée}

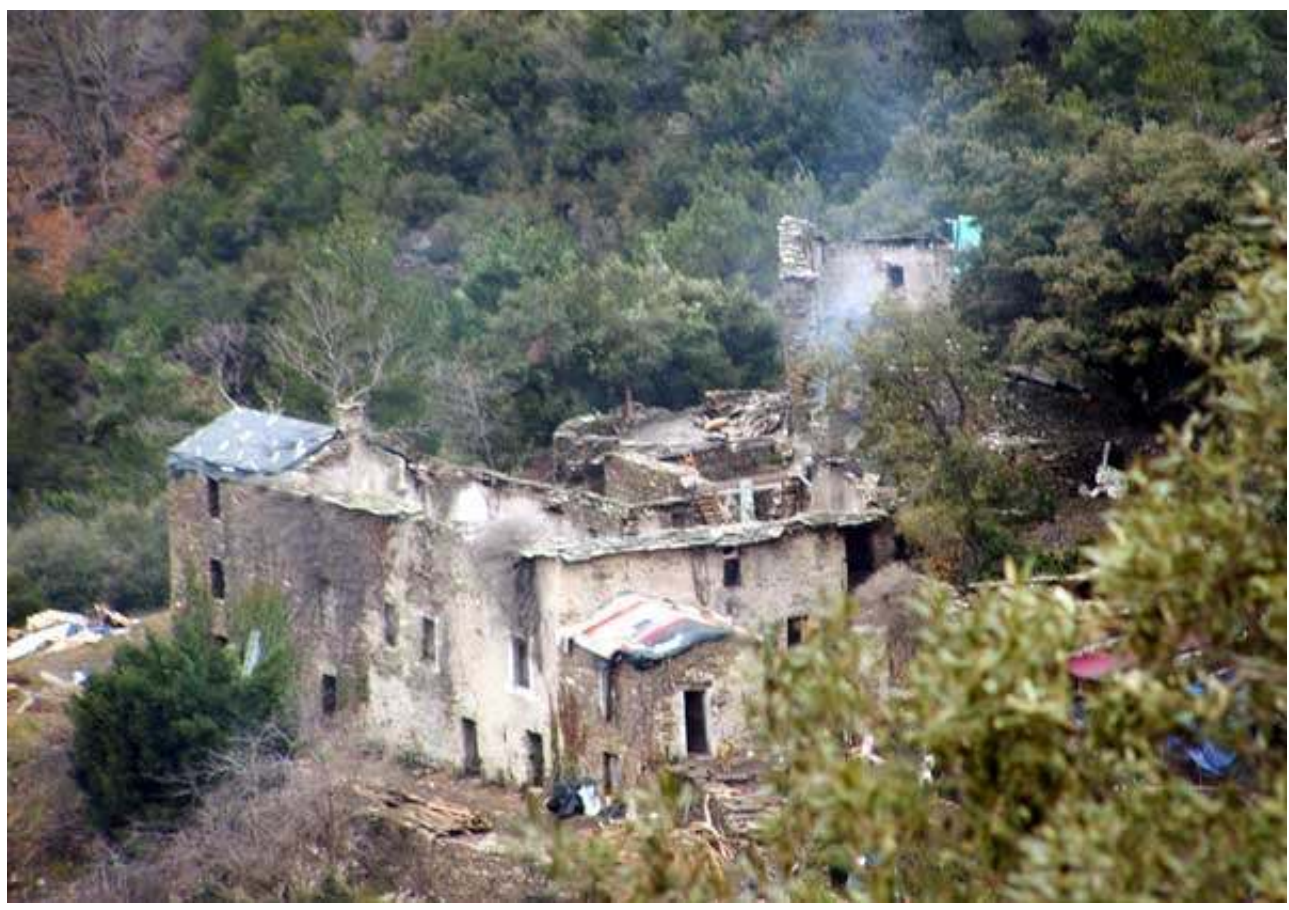

Cliché site Internet de la Picharlerie

2 «Dans cette vallée surplombée par la Corniche des Cévennes, c'est la stupeur», commente l'envoyée spéciale de Libération ${ }^{1}$. L'intensité des réactions suscitées par cette destruction est en effet à la mesure de la brutalité de l'événement: "Des personnes affluent, nous témoignant sous des formes multiples leur sympathie [...] Des élus s'en mêlent; des anciens crient leur indignation : on a détruit une partie de leur passé ", renchérissent les ex-occupants du lieu. Le conseiller général du canton se confie à un grand quotidien régional : «Je suis complètement bouleversé. Et je ne suis pas le seul [...] car la Picharlerie est un nom qui parle à toute la Lozère. » C'est en effet un haut lieu de la résistance au nazisme durant la seconde guerre mondiale que les forces de l'ordre ont rasé, un "symbole des Cévennes $»^{2}$. Pour beaucoup, cet acte, décidé par le préfet avec l'accord du propriétaire, un pasteur à la retraite, fait figure de vandalisme d'État, comme en témoigne le titre d'un pamphlet : «Un vandalisme légal : un lieu chargé d'histoire de résistances passé au bulldozer $»^{3}$. Quant à Jacques Poujol, un ancien résistant, il affirme avoir «vécu comme un cauchemar la nouvelle du passage du bulldozer à la Picharlerie [qui] illustre par excellence, non pas la Résistance en général, abstraite et inhumaine, mais les résistances au pluriel »". En 2005, la section locale du Club Cévenol, une association culturelle qui œuvre à la préservation et à la promotion du patrimoine des Cévennes, avait d'ailleurs émis le vœu d'ériger à la Picharlerie une stèle pour honorer la mémoire des maquisards.

3 La destruction de ce mas, considéré comme l'épicentre du paysage mémoriel de la Résistance et érigé en "haut lieu de mémoire ", est donc à l'origine d'une véritable déflagration émotionnelle qui a suscité localement de vifs remous ainsi qu'un puissant mouvement de protestation. Ainsi l'affaire de la Picharlerie illustre-t-elle pleinement les propos de Jacques Le Goff : le patrimoine, étroitement lié à l'histoire et à la mémoire, « est ipso facto enjeu de choix passionnels et de conflits ardents $»^{5}$. Que nous dévoile le discours 
patrimonial des rapports sociaux à l'oeuvre sur un territoire, des enjeux idéologiques, identitaires et politiques qui le sous-tendent? Pour tenter d'y répondre, nous interrogerons les significations contrastées dont est lestée la Picharlerie en tant qu'objet patrimonial ainsi que les controverses qui se sont nouées à son propos. Nous verrons ainsi que, derrière une émotion et une mobilisation qui de prime abord ont pu sembler très consensuelles, se dessinent en réalité des interprétations distinctes, si ce n'est concurrentes, du passé et du patrimoine. L'émotion patrimoniale apparaît comme étroitement corrélée à des registres de sens, des régimes de valeurs et des passions politiques qui ne revêtent ni le même contenu ni la même signification pour tous ceux qui ont cependant dénoncé d'une même voix cette destruction. Ce sont ainsi deux récits concurrents qui s'efforcent chacun d'imposer un usage légitime du passé6.

\section{La Picharlerie}

4 Pour saisir les enjeux mémoriels que cet acte de destruction a mobilisés, il y a lieu de revenir sur la place que tient la Picharlerie dans l'histoire locale.

5 Une première séquence s'inscrit dans le mouvement général de désertification rurale qui a particulièrement affecté les Cévennes de 1850 aux années 1970. La Picharlerie, un mas imposant situé sur les hauteurs de Moissac-Vallée-française est abandonné par ses propriétaires dans les années 1930, suite au déclin de la sériciculture et à la crise castanéicole.

6 Une seconde séquence se situe durant la seconde guerre mondiale. À partir de 1943, des réfractaires au service de travail obligatoire en Allemagne tentent de se cacher dans ces montagnes propices à la clandestinité. Comme ils sont jeunes et sans aucune formation militaire, deux résistants locaux décident d'y créer un maquis-école. En février 1944, le maquis Bir Hakeim s'y installe, son chef voulant concentrer l'ensemble des maquis de la région autour de la Picharlerie. En avril de la même année, les maquisards qui se dissimulent dans le secteur sont encerclés par les soldats allemands, mais parviennent à s'échapper7. En représailles, le mas est mitraillé par les SS.

7 Une troisième séquence correspond au retour de la paix. Le mas est à nouveau abandonné et ses héritiers, indivisaires, se renvoient mutuellement la charge de son entretien. En 1976, un violent incendie parachève l'œuvre du temps. Il n'en reste plus que quelques pans de murs écroulés; des arbres poussent à l'intérieur des bâtiments et les terrasses de culture s'effondrent, minées par l'érosion et rongées par la végétation. Désormais, La Picharlerie n'est qu'un témoignage parmi bien d'autres de la déprise rurale.

8 Enfin, la quatrième séquence s'ouvre au début des années 2000. Le mouvement d'installation rurale, amorcé depuis l'après-68, n'a pas faibli mais s'est même accru. De nouveaux candidats à l'installation, que la flambée des prix de l'immobilier prive de l'accession au logement et au foncier, explorent la montagne à la recherche de maisons abandonnées où s'installer. C'est l'une de ces fermes, signalée comme « ruines » sur les cartes d'État-major, qu'un groupe de personnes investit au printemps 2002, la sachant abandonnée depuis près de soixante dix ans. (ill.2) 


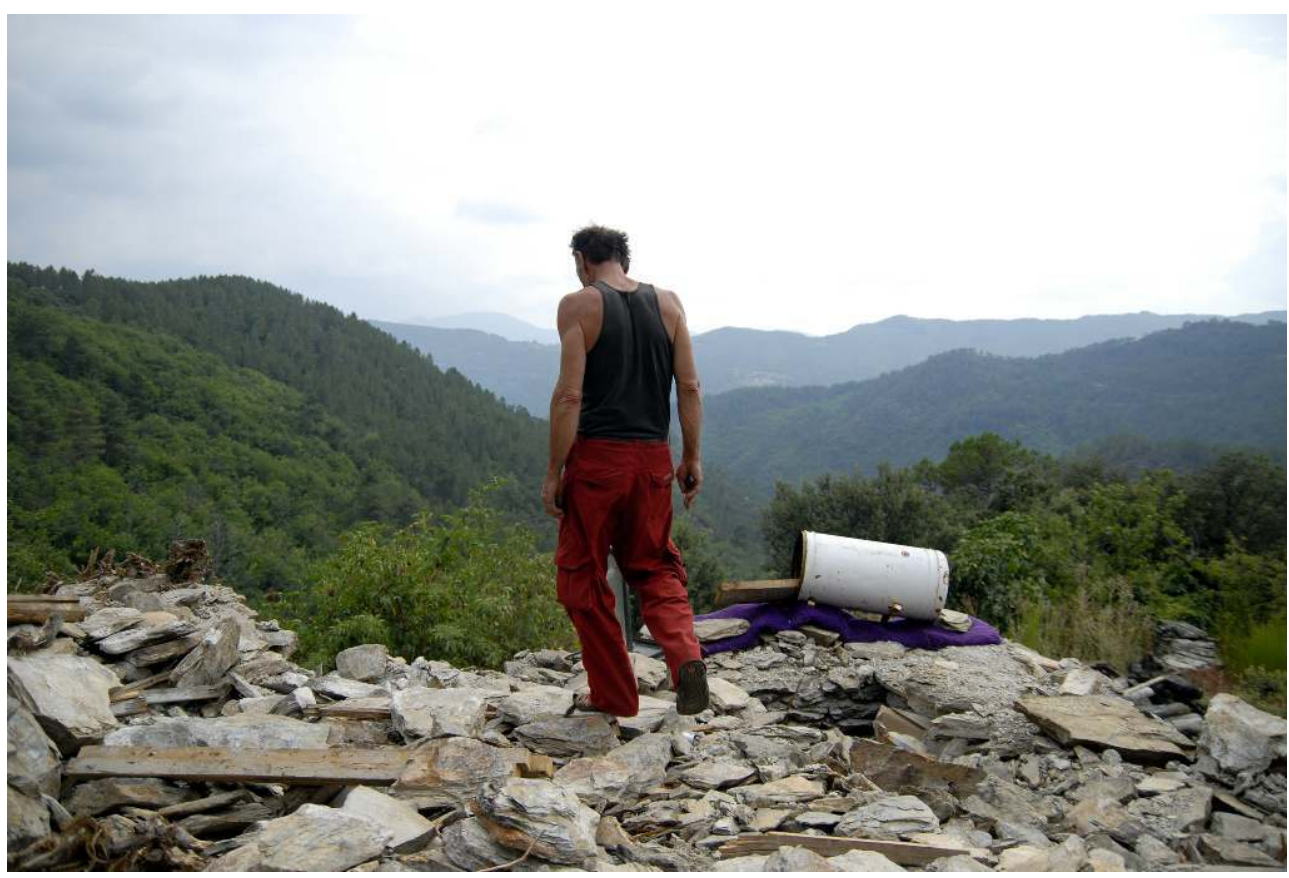

Illustration d'un article publié par le quotidien Libération le 13 août 2007

Cl. Nanda Gonzague/TransitSous l'empire de l'émotion

Dans les semaines qui suivent la démolition de la Picharlerie, les réunions se multiplient en vallée française. Le 17 juillet, Jean Bonijol, le président de l'association départementale des anciens de la Résistance, considéré localement comme le "gardien de la mémoire résistante ", assiste à une réunion qui se tient à Sainte-Croix-Vallée-française. À cette occasion, il évoque sa formation au maquis-école de la Picharlerie ainsi que les terribles combats d'avril 1944 qui ont contraint les maquisards à se disperser. La foule qui se presse est extrêmement mêlée : des habitants du village, des élus locaux, des protestants, des résistants mais aussi des militants libertaires, des néo-ruraux depuis longtemps installés dans le coin, et bien entendu les ex-occupants de la Picharlerie. Tous ont le sentiment de vivre un intense moment de partage, un même élan de révolte contre le caractère injustifiable d'une sanction qui semble disproportionnée au regard des motifs qui la fondent. Le 26 juillet, un autre rassemblement a lieu à Florac, devant la préfecture, pour protester contre l'attitude de l'État, considérant qu'au-delà de la question des occupants illégaux du mas, le but poursuivi n'est autre qu'une normalisation liberticide de toute la société. À Sainte-Croix-vallée-française, le 25 août suivant, les squatters expulsés prennent l'initiative d'une réunion. Mais contrairement à ce qui s'est passé un mois plus tôt, on ne compte plus d'anciens résistants ni de Cévenols d'origine parmi les présents. Quant aux deux seuls élus qui s'y sont joints, issus l'un comme l'autre du milieu néorural, ils affirment être là à titre personnel.

10 Si on observe l'évolution de la participation à ces diverses rencontres protestataires, on constate donc qu'au fil des semaines, le profil sociologique des participants a sensiblement évolué. De profondes divergences de vue ont surgi quant aux significations qui seraient attachées à ce lieu de mémoire. Au-delà du sentiment partagé d'une perte irréparable, la destruction de la Picharlerie a suscité de si profonds antagonismes au sein de la population que certains élus s'inquiètent de l'incidence de l'événement sur la fragile 
paix sociale qui règne dans la Vallée française. Le président de la communauté de communes a beau appeler au calme et au dialogue, rappeler dans une délibération largement diffusée que, sans la participation active des nouveaux habitants, les vallées seraient « dans un état de friche et de désertification » mais qu'inversement sans l'accueil qu'ils ont reçu et sans la transmission des savoir-faire anciens ces derniers n'auraient pu rester, la tension entre les habitants ne cesse de s'accentuer.

\section{La Résistance patrimonialisée : la mobilisation du passé}

11 Dans un précédent travail ${ }^{8}$, m'attachant à identifier ce qui en Cévennes fait patrimoine, j'ai montré que l'identité imaginée s'y est notamment déployée à partir du modèle du camisard. L'importance du mouvement de résistance au nazisme durant la seconde guerre mondiale n'a fait que le confirmer, avec l'assimilation récurrente entre camisard et maquisard. Parmi les motifs qui fondent le discours local sur le passé et sur l'identité, l'esprit de résistance apparaît donc comme l'un des plus fédérateurs. Quelle famille cévenole, en effet, ne possède pas son camisard, son galérien pour la foi ou son maquisard, autrement dit ne se situe pas dans la descendance d'un résistant, à la fois victime et héros? Ce sont ces modestes mais prestigieux ancêtres qui confèrent à chaque chaîne familiale " une raison supérieure d'être, puisque son sang et son sens sont sacrés par l'histoire $»^{9}$. De toutes ces mémoires individuelles émerge ainsi une mémoire collective qui confère au discours identitaire son contenu et sa prégnance affective.

ristance - à la fois valeur et moment historique - est donc reconnue par la population locale comme une composante majeure de son identité et de son patrimoine. Elle est le fil conducteur qui relie le présent au passé, les luttes contemporaines au maquis de la deuxième guerre mondiale et, plus loin encore dans le passé, au Désert de la révolte des camisards.

Un hymne du maquis composé par Jacques Poujol alors qu'il était un jeune chef de groupe du maquis Aigoual-Cévennes établit d'ailleurs clairement la continuité, il est vrai facilitée par la rime et le quasi anagramme :

«Les fiers enfants des Cévennes,

Réfractaires et maquisards,

Montrent qu'ils ont dans les veines

Le sang pur des Camisards »

14 C'est lui également qui rapporte que Lucie Aubrac, Cévenole d'adoption à laquelle il rend hommage, rattachait l'origine du mot Résistance « au mot d'ordre que les Cévenols n'ont jamais oublié, celui que Marie Durand grava dans la pierre de la tour de Constance ». La continuité entre ces deux temps forts de l'histoire locale est également signifiée par le titre que Muse Darblay et Tristan Sévère ont donné à leur ouvrage paru en 1944 : Des camisards aux maquisards. Cette identification est même essentialisée par les animateurs d'un site consacré aux camisards : la filiation ente les deux termes serait selon eux « toute naturelle $»^{10}$.

L'esprit de résistance censé caractériser la mentalité cévenole, cet " esprit frondeur » dans lequel la population aime à se reconnaître, ne sont pas sans attrait pour tous ceux qui s'y sont installés à diverses époques. Selon un dossier de l'Académie de Montpellier consacré aux Cévennes ${ }^{11}$ et intitulé « Terre de résistances », il aurait également séduit les 
néo-ruraux des années 1970 et, plus récemment, les alter-mondialistes, « résistants eux aussi ", qui ont trouvé en Cévennes " un havre de paix et aussi de combat ». Un point de vue que partagent les occupants de la Picharlerie: pour eux, l'histoire des Cévennes «s'est à plusieurs reprises identifiée, au cours des siècles, à l'histoire des résistances aux pouvoirs en place, jadis royal et religieux, hier collaborationniste, plus récemment au mode de vie dévastateur et inhumain du monde moderne ».

Mais la résistance ne revêt pas qu'une signification locale, elle appartient également à la mémoire collective du pays tout entier, au « Panthéon des références sacrées de la nation française $»^{12}$. Substantivée, elle occupe dans l'imaginaire national une place majeure car elle véhicule les valeurs cardinales de la nation française : fraternité, justice et tolérance. La figure du Résistant incarne donc une certaine conception de l'identité nationale, adossée à des valeurs morales. Elle crée ainsi un espace social commun, susceptible de rassembler la population de l'Hexagone dans ce que Jean-Yves Boursier appelle une «topolâtrie mémorielle $»^{13}$.

\section{Une mémoire disputée, entre accord patrimonial et conflit de légitimité}

17 Même si l'émotion suscitée par l'événement, en tant que comportement collectif, se manifeste de manière homogène et s'exprime dans des termes sensiblement identiques, son vocabulaire étant relativement restreint, une lecture univoque de celle-ci serait réductrice. Mobilisée différemment selon les personnes et les groupes, elle reflète des visions $\mathrm{du}$ monde distinctes, voire concurrentes. Ainsi les tensions qui se font progressivement jour au sein de la population locale, une fois le pic émotionnel passé, sont liées au décalage entre leurs horizons d'attente et d'expérience respectifs.

18 Au-delà de l'affectivité qui imprègne et informe les discours et les actes, la réaction collective est en effet formée d'idéaux et d'attachements divers à des symboles qui ne revêtent pas la même signification pour chacun. Ce sont en particulier deux lectures de la Résistance patrimonialisée qui s'affrontent, validant ainsi la remarque de Patrice Béghain selon laquelle le patrimoine présenterait désormais un double visage : celui de l'essence, de l'identité, et celui de l'être, du projet et de l'action ${ }^{14}$.

19 L'enquête montre que deux discours antagonistes tentent chacun d'imposer leur usage légitime du passé et leur définition de la Picharlerie comme bien commun.

20 Le premier est généralement le fait de personnes - principalement des Cévenols d'origine - qui revendiquent leur autochtonie en défendant une conception essentialiste et exclusive de l'identité. Le lieu patrimonial est perçu comme la matérialisation de l'histoire locale, témoin d'une identité enracinée, d'une appartenance fondée sur la parenté et la transmission de la mémoire familiale. Dans cette perspective, la Picharlerie apparaît comme le réceptacle d'une identité figée sur l'énonciation de son passé : dès lors, la destruction de ce «lieu mythique » est ressentie comme un véritable déni d'identité. L'esprit de résistance, naturalisé, renvoie à une conception quasi génétique de l'appartenance qui n'est pas sans conséquences; la rhétorique identitaire procède dans ce cas d'un modèle qui se moule volontiers dans le communautarisme et favorise l'intolérance. De ce point de vue, les squatters incarnent une double menace: non seulement ils sont étrangers à la région et ne sont donc pas issus d'une lignée résistante locale qui leur conférerait une légitimité, mais en outre leur positionnement politique est 
source d'inquiétude et d'incompréhension. La mémoire collective apparait donc bien comme « un discours de l'altérité où la possession d'une histoire qu'on ne partage pas donne au groupe son identité $»^{15}$; on comprend mieux alors les réticences des autochtones à ce que d'autres qu'eux revendiquent cette histoire : l'appropriation du symbole de la Résistance leur apparaît à la fois comme un détournement de sens et comme une dépossession.

Le second discours, porté par les ex-squatters et par ceux qui les soutiennent, est en rupture avec le précédent. Contre une conception fermée du patrimoine et de l'identité que ce dernier véhicule, il suggère au contraire que l'occupation de la Picharlerie a été une manière de faire vivre l'héritage en l'actualisant. Il n'est donc pas innocent que le terme de «lieu de mémoire » soit préféré par les squatters à celui de patrimoine : ainsi que le fait remarquer Marc Augé, les lieux de mémoire existent pour autant que la mémoire dont ils participent demeure vivante, constitutive du présent ${ }^{16}$ (1989); or, précise Mona Ozouf, donner la parole au présent ne consiste pas à voir en lui l'héritier du passé mais plutôt son usager, toujours susceptible, ajoute-t-elle, « de ranimer des objets qui somnolent $\aleph^{17}$. Dans cette appropriation patrimoniale, on peut également voir une quête de reconnaissance : l'universalité des valeurs incarnées par le lieu n'offre-t-elle pas une voie détournée pour se doter d'une légitimité au regard du territoire, en suggérant que les «bons fils » seraient moins les héritiers «biologiques » que ces nouveaux venus qui font preuve non seulement d'une connaissance de l'histoire locale mais aussi d'une volonté de sauvegarder cet héritage?

\section{La concurrence des patrimoines}

Ce sont donc bien deux lectures du patrimoine qui sont en concurrence. La première, classique, proche du modèle originel, y voit un bien transmis au sein d'un collectif selon l'axe des générations. Fondamentalement conservatrice, cette politique de la mémoire est exclusivement tournée vers une reconstruction/idéalisation du passé. La seconde renverse au contraire le rapport au passé en s'inscrivant dans la perspective ouverte par Jean Davallon avec sa notion de "filiation inversée " qui renvoie à une construction «depuis le présent» de la filiation, nous instituant ainsi « héritiers de ces choses du passé $»^{18}$. "Qui peut prétendre à vivre dans ce pays?", s'interroge une personne qui soutient les occupants: "Ceux qui ne l'ayant pas reçu en cadeau d'héritage le font renaître en y habitant, en le restaurant à leur manière, en le cultivant, ou bien celui qui l'ayant reçu de ses ancêtres le tue en l'abandonnant puis en le rasant ?» C'est également l'avis d'un mystérieux « comité du Bois » qui réagit sur le site Internet de la Picharlerie : « 50 ans après, qui est là ? Qui reconstruit le toit parti en fumée, les étages? Qui reprend les traversiers aux griffes des ronciers? Qui rouvre les circuits subtils de l'eau? Qui redonne vie à cette âme morte qui a tant vécu ? Des gens qui inventent une vie nouvelle au lieu de jouer les victimes. » 
III. 3 : Ruines avec guitare fracassée en premier plan

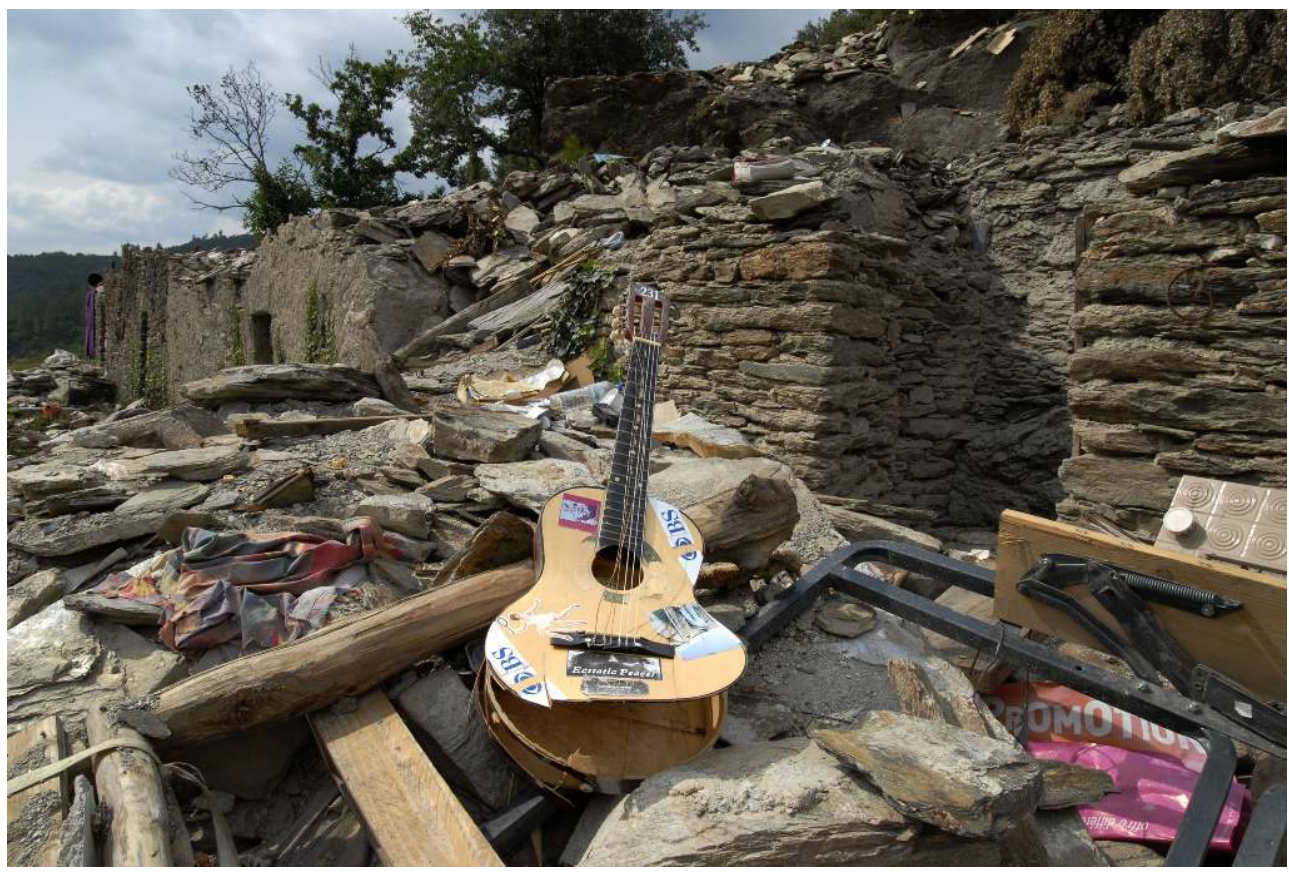

" On maraude mes pommes, j'arrache le pommier » (G. de Maupassant). Titre d'une déclaration collective en réaction à la destruction de la Picharlerie.

Cl. Nanda Gonzague/Transit

23 Les ex-occupants s'engouffrent dans la brèche ouverte à la fois par le statut du propriétaire (un pasteur!) et par le discours identitaire d'une partie de la population locale. Ils condamnent l'attitude du pasteur « obscurantiste » ainsi que de tous ceux qui selon eux préfèrent encore voir la Picharlerie « rasée et morte, plutôt qu'occupée et vivante ». Le fait qu'un pasteur porte principalement la responsabilité de cet acte destructeur est en effet vécu comme un véritable séisme. Il provoque une profonde incompréhension tant l'acte est en contradiction avec les valeurs de tolérance et de solidarité que revendique le protestantisme : "Voilà un serviteur de Dieu, et qui plus est de l'Église réformée de France pour laquelle [...] l'accueil des réprouvés du monde et la notion de résistance à l'oppression ne sont pas des vains mots, qui n'aura pas assez d'années désormais pour mettre en cohérence ses actes et ses convictions » remarque un des soutiens des squatteurs; un autre n'a pas de mots assez durs pour fustiger ce serviteur de la religion réformée «qui a préféré fouler aux pieds ce pour quoi il était appelé ». Le malaise provoqué par son attitude est d'autant plus vif que L'Église réformée est souvent allée extrêmement loin dans son souci d'actualisation du passé ${ }^{19}$. Un engagement que le propriétaire de la Picharlerie ne saurait ignorer, pas plus qu'il ne méconnaît le fait que la minorité protestante est indissociable, par la résistance dont elle a fait preuve face au rejet et aux persécutions, de l'idée de liberté ; c'est au nom de celle-ci que les ex-occupants de la Picharlerie dénoncent la visée liberticide qu'ils décèlent dans la décision du préfet de procéder à la destruction du lieu. Le « comité du bois " s'indigne d'ailleurs de la faible réaction du milieu protestant alors que l'évènement aurait dû avoir un retentissement tout particulier dans une population qui se flatte d'avoir joué un rôle majeur dans l'institution de valeurs qui ont été fixées dans le marbre de la déclaration des droits de l'homme et du citoyen : «Faut-il rappeler les bancs de nage des galères auxquels ils étaient enchaînés parce qu'ils avaient exercé cette liberté de pensée ? Et l'inscription 
« résister " gravée dans la pierre du cachot de la tour de Constance à Aigues-Mortes?» Pour eux, il importe donc qu'« au souvenir lâche et futile, le présent oppose et propose ses luttes ».

III. 4 : Tract en noir et blanc

Apres la destruction au bulldozer (soutenue par la préfecture de la Lozére) de la Picharlerie. haut-lieu de mémoire de la résistance au naxisme et occupé depuis 5 ans :

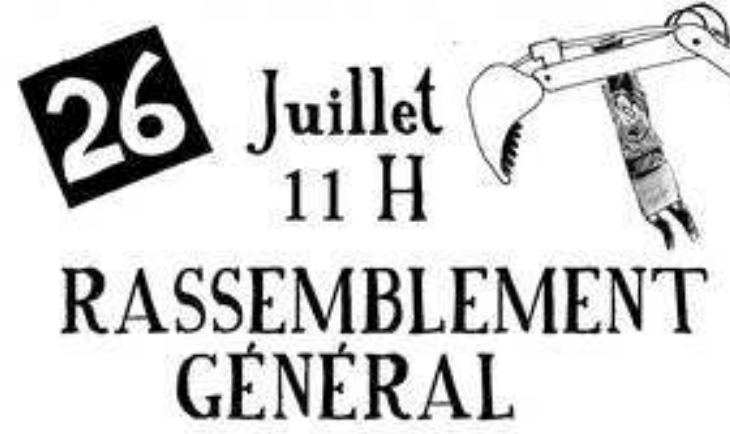

\section{devant la sous-préfecture de Florac (48)}

Apportez vos idées - textes - grogne - musique - cri - danse - pancartes - cairn vol au vent - tarte à la créme - plat de résistance...

Mangeons ensemble plutot que détre mange tout seul dans son coin : Pic-nic festif

Tract annonçant un rassemblement protestataire et de soutien aux expulsés

Cl. Françoise Clavairolle

\section{La politisation du débat : entre localité et universalité}

Insensiblement, de réunions en forums de discussion, l'émoi, qui immédiatement après la démolition de la Picharlerie était de nature patrimoniale, revêt une résonance nettement plus politique. Le rassemblement du 25 août 2007 marque à cet égard un tournant : alors qu'un débat houleux met aux prises les partisans d'un dialogue avec la population autochtone et les squatters qui depuis l'événement sont en voie de radicalisation, la création d'un collectif "pour vivre et résister ici et maintenant» est annoncée. Ses objectifs sont présentés dans un texte illustré par un dessin qui est une allusion directe à la destruction de la Picharlerie : une pelle mécanique dont le bras est armé d'une masse utilisée pour les démolitions.

Dès lors, les divergences ne vont plus cesser de se creuser, entre les squatters et les Cévenols d'origine dans un premier temps, puis au sein même du réseau des squatters.

Ce sont d'abord les anciens résistants qui se désolidarisent des squatters, se refusant à comparer « l'illégalité du squat avec l'illégalité de ceux qui ont risqué leur vie en 39-45 $»^{20}$ . Puis ce sont des évènements conçus dans le but de re-souder la population qui tournent court, comme la randonnée commémorative à la Picharlerie organisée en avril 2008 en remplacement de la pose de la stèle ; loin de calmer les esprits, elle ne fait au contraire qu'aviver les dissensions car le mot d'ordre des organisateurs, « oublier les polémiques du XXI ${ }^{e}$ siècle » - allusion à peine voilée à la controverse provoquée par la destruction de la Picharlerie -, est aussitôt traduit lapidairement par un proche des anciens squatters : « La 
résistance commence avec les héros et les martyrs du comité national de la Résistance... et s'y arrête. Ainsi béatifiée, l'histoire se fige.»

Mais des désaccords surgissent également entre les squatters et leurs sympathisants, comme le montre la lettre ouverte qui leur est adressée par un ancien militant maoïste, familier de la région, dans laquelle il met en garde contre «les dangers de la pente savonneuse sur laquelle [ils] se sont engagés en reprenant à leur compte les mythes de la Résistance ». Y voyant au contraire le prélude à la reconstitution de l'État-nation, ce qui est loin à ses yeux de satisfaire la volonté d'autonomie affiché par les squatters, il les exhorte à ne pas venir «bassiner avec la Résistance, terme qui renferme le même mensonge que celui de démocratie $»^{21}$.

Deux ans après le rasement de la Picharlerie, la sensibilité à l'événement est toujours aussi vive, mais les significations qui y sont attachées se sont progressivement déplacées. La polémique qui s'est nouée autour du sens qu'il convient de conférer à ce haut lieu de la Résistance a été le levier dans un premier temps d'une crise locale des rapports sociaux, puis d'une mobilisation politique, proche des nouveaux mouvements sociaux et visant en quelque sorte à « délocaliser» le discours local ${ }^{22}$. Tandis que les « locaux» prennent de plus en plus leurs distances avec les squatters, leur reprochant d'instrumentaliser la Résistance afin de légitimer une occupation illégale et qui porte atteinte au droit de propriété, ces derniers dénoncent en retour une pratique de la mémoire volontairement sélective et déformante, qui n'aurait d'autre objectif que de justifier leur exclusion.

On peut se demander dans quelle mesure ce n'est pas l'impossibilité à dépasser cette opposition entre deux usages du passé qui aurait conduit à creuser la frontière entre d'un côté les squatters qui, à la faveur de l'événement se sont organisés en collectif, et d'autre part les autochtones qui y puisent une confirmation d'identité. La radicalisation de leurs dissensions a eu pour conséquence l'émergence d'un mouvement social qui s'est progressivement affranchi de la référence au passé pour revendiquer non plus une filiation verticale, mais une fraternité horizontale entre et avec les victimes d'une injustice sociale prenant sens à l'échelle mondiale tout en s'actualisant localement.

Le concept même d'« émotion patrimoniale » mérite d'être interrogé à la lumière de cette étude de cas. En se focalisant en effet sur la dimension patrimoniale des affects, ne courton pas le risque d'une lecture simplificatrice de ces mobilisations collectives? En effet, elles ont pour caractéristiques de n'être que rarement " pures ", puisqu'elles sont tout à la fois spontanées et instrumentalisées, et d'être également dynamiques, dans la mesure où le jeu des rapports sociaux génère des glissements interprétatifs, des réappréciations qui re-signifient le lieu. Dans le cas étudié, la frontière semble extrêmement tenue et poreuse entre la réaction collective engagée au nom du patrimoine et celle qui l'est au nom d'une certaine conception de la justice sociale dont Daniel Céfaï a bien montré dans son récent ouvrage sur l'action collective qu'elle était également pourvoyeuse d'émotions ${ }^{23}$. 


\section{NOTES}

1. . Carole Rap, « Ci-git "La Pich" et le souvenir du maquis », Libération, 13 août 2007.

2. . Midi-Libre, 21 juillet 2007.

3. Selon Dominique Poulot, le discours patrimonial est né de l'effroi face au vandalisme.

4. Jacques Poujol, «La Picharlerie. Un carrefour mouvementé des résistances », Patrimoine 30, $\mathrm{n}^{\circ}$ 19, 2007, p.19.

5. . Jacques Le Goff (dir.), Patrimoine et passions identitaires, actes des entretiens du patrimoine, t. III, Paris, Fayard-CNMHS/éd. du Patrimoine, 1998, 445 p.

6. . Maryline Crivello et Nicolas Offenstadt, «Introduction », Maryline Crivello, Patrick Garcia et Nicolas Offenstadt (éd.), Concurrence des passés. Usages politiques du passé dans la France contemporaine, Aix-en-Provence, presses universitaires de Provence, 2006, p. 191-202.

7. . Jacques Poujol, « La Picharlerie... », op. cit., p. 23.

8. . Françoise Clavairolle, La Borie sauvée des eaux. Ethnologie d'une émotion patrimoniale, Rapport à la mission à l'ethnologie, direction de l'architecture et du patrimoine, ministère de la culture, 2006, $246 \mathrm{p}$.

9. . Jean-Noël Pelen, « Le légendaire de l'identité communautaire en Cévennes, du XVIIIe siècle au XXe siècle », Le Monde alpin et rhodanien, $\mathrm{n}^{\circ} 1-4,1982$, p. 130.

10. . http://www.camisards.net.

11. Académie de Montpellier, «Terres de résistance. Les maquis des Cévennes gardoises (1942-1945)», http://www.ac-montpellier.fr/ia30/actualites/actuas_2005/documents/ cevennes_resistance.pdf

12. . Philippe Hanus, « Mémoire, histoire et actualité de la Résistance. Vassieux-en-Vercors. 16 octobre 2004 ", Bulletin de l'Association Rhône-Alpes d'Anthropologie, n54, 2005, p.12.

13. . Jean-Yves Boursier, «Les enjeux politiques des "musées de la résistance". Multiplicité des lieux », L'Esprit des lieux : le patrimoine et la cité, Grenoble, presses universitaires de Grenoble, 1997, p. 287

14. . Patrice Béghain, Le Patrimoine : culture et lien social, Paris, presses de sciences po., 1998, p. 102. 15. . Françoise Zonabend, La Mémoire longue, Paris, presses universitaires de France, 1980, p. 310 . 16. . Marc Augé, «Les lieux de mémoire du point de vue de l'ethnologue », Gradhiva, n6, 1989, p. 3-12.

17. Mona Ozouf, citée par Dominique Poulot, Patrimoine et musées. L'institution de la culture, Paris, Hachette, 2001, p. 202.

18. . Jean Davallon, « Le patrimoine : « une filiation inversée »?», EspacesTemps, n74-75, 2000, p. 11.

19. . À l'occasion de l'assemblée du musée du Désert de 1942, le pasteur Boegner a invité les pasteurs présents à tout mettre en œuvre pour sauver des juifs (Philippe Joutard, «Le Musée du Désert : une mémoire protestante ", Pierre Nora, (dir.), Les lieux de mémoire I, les France III, Paris, Gallimard, 1992, p. 2673-2675.

20. . Carole Rap, « Ci-gît », op. cit.

21. André Dréan, « Des mythes de la Résistance en Cévennes et ailleurs », A-Infos, 2007, http:// ainfos.ca/fr/ainfos06913.htlm

22. . Maryline Crivello et Nicolas Offenstadt, "Introduction », op. cit., p. 199.

23. . Daniel Céfaï, Pourquoi se mobilise-t-on? Les théories de l'action collective, Paris, La Découverte/ MAUSS, 2007, $730 \mathrm{p}$. 


\section{RÉSUMÉS}

Dans les Cévennes, la destruction d'un « haut lieu de mémoire » de la Résistance dans le but d'en déloger un collectif d'occupants illégaux a déclenché au sein de la population une vive réaction émotionnelle. L'article s'attache à interroger les représentations du passé et du patrimoine qui ont été mobilisés par tous ceux qui ont protesté contre cet acte, qualifié par certains de «vandalisme d'État ». Il questionne les enjeux de mémoire et montre que l'émotion patrimoniale a été le levier d'une crise locale des rapports sociaux.

In the Cevennes, a 'haut lieu de mémoire', an important place of memory associated with the Resistance during the Second World War, was destroyed in order to evict a collective of illegal occupants. Within the local population, this destruction had strong emotional repercussions. This article attempts to interrogate the different representations of the past and of the heritage which were mobilised by those who protested against the act of demolition, qualified by some as 'State vandalism'. It questions issues of memory and shows how this heritage emotion was the catalyst for a local crisis in social relations.

In den Cevennen löste die Zerstörung eines haut lieu de mémoire (besondere Gedenkstätte) für die Résistance aus dem Grunde, ein Kollektiv illegaler Besetzer zu vertreiben, eine heftige emotionale Reaktion unter der Bevölkerung aus. Der Artikel nimmt sich der von den Protestierenden aufgebrachten Vorstellungen der Vergangenheit und des Kulturerbes an, um diese Tat als " vandalisme d'état" zu bezeichnen. Er untersucht die Zielsetzungen des Gedenkens und zeigt, wie die émotions patrimoniales einer lokalen Krise der sozialen Beziehungen Vorschub leisteten.

\section{INDEX}

Index chronologique : époque contemporaine

Schlüsselwörter : Kulturerbe, Erinnerungsort

Mots-clés : lieu de mémoire, patrimoine

Keywords : memory space, heritage

\section{AUTEUR}

\section{FRANÇOISE CLAVAIROLLE}

Françoise Clavairolle est née en 1953. Elle est maîtresse de conférences en anthropologie à l'université François-Rabelais de Tours et membre de l'équipe « Construction politique et sociale des territoires » (COST), UMR CITERES - CNRS/université François-Rabelais. Dernier ouvrage paru : Le renouveau de la production de la soie en Cévennes (1972-1998), Tours, presses universitaires François-Rabelais, 2008.

Adresse électronique : Francoise.clavairolle@univ-tours.fr 